\title{
Baryon Mass in medium with Holographic QCD
}

\author{
Yunseok Seo* and Sang-Jin $\operatorname{Sin}^{\dagger}$ \\ Department of physics, BK21 Program Division, \\ Hanyang University, Seoul 133-791, Korea
}

\begin{abstract}
We study the baryon vertex (BV) in the presence of medium using DBI action and the force balance condition between $\mathrm{BV}$ and the probe branes. We note that a stable BV configuration exists only in some of the confining backgrounds. For the system of finite density, the issue is whether there is a canonical definition for the baryon mass in the medium. In this work, we define it as the energy of the deformed BV satisfying the force balance condition (FBC) with the probe brane. With FBC, lengths of the strings attached to the BV tend to be zero while the compact branes are enlongated to mimic the string. We attribute the deformation energy of the probe brane to the baryon-baryon interaction. We show that for a system with heavy quarks the baryon mass drops monotonically as a function of density while it has minimum in case of light quark system.
\end{abstract}

*yseo@hanyang.ac.kr

${ }^{\dagger}$ sjsin@hanyang.ac.kr 


\section{INTRODUCTION}

Recently there has been much interest in studying QCD in terms of AdS/CFT correspondence [1]. One particularly interesting question is the study of nucleon in the dense matter system. Baryons in AdS/CFT was first introduced by Witten in [2], where baryon is identified with a compact D-brane wrapping the directions transverse to the $N_{c}$ color Dbranes with $N_{c}$ strings attached to it. The baryon charge is carried by the end point of the strings connecting color and flavor branes. After the chiral model of Sakai-Sugimoto[3, 4] was developed, baryon was discussed as the instanton on the probe branes [5-8] and effective field theory of baryon was developed in [9] in the context of phenomenological AdS/QCD model [10].

The baryon density problem was first discussed in $[11,12]$ where chemical potential is identified as the asymptotic value of the electric potential on the flavor brane. The D3/D7 system of finite temperature and finite baryon density was analyzed in [13] where both Minkowski embedding as well as the black hole embedding were considered. However, it has been argued [14] that in the presence of the quark density the D7 brane has to touch the black hole horizon because the strings connecting D7 and the horizon can be replaced by the spiky deformation of the D7 brane. So it is an interesting to ask what happens if there are baryons not just quarks.

In the presence of baryon vertex (outside the black-hole horizon), the string would not touch the horizon and there would be no reason for the spiky D-brane to touch the horizon. See figure 1-a. The issue is then whether there is a baryon vertex [2] for the given background and given probe branes. The probe branes are pulling the baryon vertex to sustain it from the gravitational pulling of the core, and it is not obvious why a compact D-brane does/doesn't exist in such background a priori. See figure 1b. If a BV configuration does not exist in the black-hole background, it is not clear whether it exist even at the confining background. This question is even more compelling since in D4-D8 $\bar{D} 8$ setup, a baryon vertex can exist regardless whether D4 background is in the confining or deconfining phase [15]. One of the purpose of this paper is to examine this issue in various gravity backgrounds with the probe

probe branes using the equation of motion and Force Balancing Condition (FBC). We will find that there exist baryon vertex $(\mathrm{BV})$ only in some of the confining backgrounds, while no such object in any of the black hole backgrounds. 


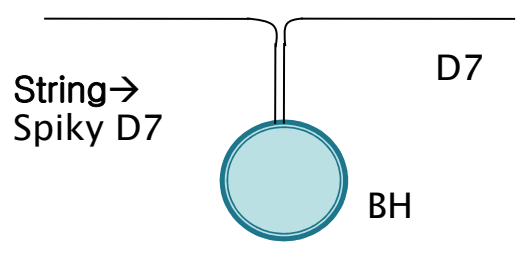

a) with quarks

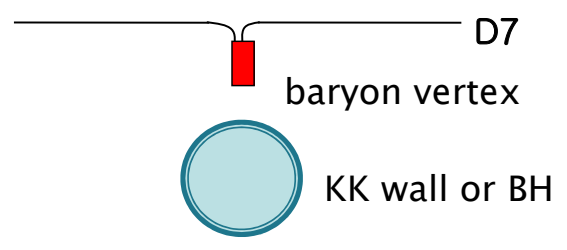

b) with baryons

FIG. 1: Black hole embedding in quark phase v.s Minkowski Embeddings in baryon phase

In case there are baryons, interesting question is the density dependence of the baryon mass when baryon vertex exists. Here the central issue is whether there is any canonical definition of mass in the absence of Lorentz symmetry. In field theory, to separate the baryon mass in the medium from the inter-baryon interaction energy has ambiguity. However, in the BV picture, one can define the baryon mass in the medium simply as the energy of deformed BV and we can attribute the deformation of the the probe brane to the BaryonBaryon interaction. We show numerically that mass drops as density increases for baryons with heavy quarks, while mass v.s density profile has a minimum for baryons with light quarks. The latter behavior is shared by the systems D4/D6 and D4/D8D 8 .

The rest of the paper goes as follows. In section 2, we study numerically to show that in the black hole background, there is no compact D-brane as a solution of a DBI action. In section 3, we show that there are solutions for the baryon vertices in some confining backgrounds and study the density dependence of the baryon mass. Here we give a full details for the D4/D6 system and leave other cases to the appendix. We conclude in section 4.

\section{ABSENCE OF BARYON VERTEX IN THE DECONFINING BACKGROUND}

In this section, we consider D6 probe branes embedded in the non-extremal black D4 background and show that the solution of a DBI action for the baryon vertex satisfying the force balance condition does not exist. We discuss D3/D7 system briefly in the Appendix (Surprisingly, the result is not completely parallel). We start from the gravity background 
describing the deconfined phase in Euclidean signature with imaginary potential is given by

$$
\begin{aligned}
d s^{2} & =\left(\frac{U}{R}\right)^{3 / 2}\left(f(U) d t^{2}+d \vec{x}^{2}+d x_{4}^{2}\right)+\left(\frac{R}{U}\right)^{3 / 2}\left(\frac{d U^{2}}{f(U)}+U^{2} d \Omega_{4}^{2}\right) \\
e^{\phi} & =g_{s}\left(\frac{U}{R}\right)^{3 / 4}, \quad F_{4}=\frac{2 \pi N_{c}}{\Omega_{4}} \epsilon_{4}, \quad f(U)=1-\left(\frac{U_{0}}{U}\right)^{3}, \quad R^{3}=\pi g_{s} N_{c} l_{s}^{3} .
\end{aligned}
$$

Here we compactify the time $t \sim t+\beta_{t}$ to consider thermal system and we also compactify $x_{4}$ for the dimensionality by $x_{4} \sim x_{4}+\beta_{4}$ and give antiperiodic boundary condition for the fermions to break the SUSY completely. There is a horizon at $U=U_{0}$, and the Hawking temperature is given by

$$
T=\frac{1}{\beta_{\tau}}=\frac{U_{0}}{\pi R^{2}}, \quad f(U)=1-\frac{U_{0}^{3}}{U^{3}} .
$$

Introducing a dimensionless coordinate $\xi$ by $\frac{d \xi^{2}}{\xi^{2}}=\frac{d U^{2}}{U^{2} f(U)}$, the background geometry becomes

$$
d s^{2}=\left(\frac{U}{R}\right)^{3 / 2}\left(f(U) d t^{2}+d \vec{x}^{2}+d x_{4}^{2}\right)+\left(\frac{R}{U}\right)^{3 / 2}\left(\frac{U}{\xi}\right)^{2}\left(d \xi^{2}+\xi^{2} d \Omega_{4}^{2}\right)
$$

where $U$ and $\xi$ are related by

$$
\left(\frac{U}{U_{0}}\right)^{3 / 2}=\frac{1}{2}\left(\xi^{3 / 2}+\frac{1}{\xi^{3 / 2}}\right), \text { and } f=\left(\frac{1-\xi^{-3}}{1+\xi^{-3}}\right)^{2} \equiv \frac{\omega_{-}^{2}}{\omega_{+}^{2}}
$$

A baryon in the three-dimensional theory corresponds to the D4 brane wrapped on an $S^{4}$ on which $N_{c}$ fundamental strings terminate. In this configuration, the background four-form field strength can couple to the world volume gauge field $A_{(1)}$ via Chern-Simons term.

The metric (3) can be written as

$$
d s^{2}=\left(\frac{U}{R}\right)^{3 / 2}\left(f d t^{2}+d \vec{x}^{2}+d x_{4}^{2}\right)+R^{3 / 2} \sqrt{U}\left(\frac{d \xi^{2}}{\xi^{2}}+d \theta^{2}+\sin ^{2} \theta d \Omega_{3}^{2}\right)
$$

We take $\left(t, \theta_{\alpha}\right)$ as world volume coordinates of a compact D4 brane, and turn on the $U(1)$ gauge field on it to have $F_{t \theta} \neq 0$. As the ansatz for the embedding of compact D4, we assume the $S O(4)$ symmetry so that position of D4 brane and gauge field depend only on $\theta$ i.e. $\xi=\xi(\theta), A_{t}=A_{t}(\theta)$, where $\theta$ measure the polar angle of $S^{4}$ from the north pole. The 
induced metric on D4 brane is

$$
d s_{D 4}^{2}=\left(\frac{U}{R}\right)^{3 / 2} f(U) d t^{2}+R^{3 / 2} \sqrt{U}\left[\left(1+\frac{\xi^{\prime 2}}{\xi^{2}}\right) d \theta^{2}+\sin ^{2} \theta d \Omega_{3}^{2}\right],
$$

where $\xi^{\prime}=\partial \xi / \partial \theta$. The DBI action for single D4 brane with $N_{c}$ fundamental string can be written as

$$
\begin{aligned}
S_{D 4} & =-\mu_{4} \int e^{-\phi} \sqrt{\operatorname{det}\left(g+2 \pi \alpha^{\prime} F\right)}+\mu_{4} \int A_{(1)} \wedge G_{(4)} \\
& =\tau_{4} \int d t d \theta \sin ^{3} \theta\left[-\sqrt{\frac{\omega_{-}^{2}}{\omega_{+}^{2 / 3}}\left(\xi^{2}+\xi^{\prime 2}\right)-\tilde{F}^{2}}+3 \tilde{A}_{t}\right] \\
& =\int d t \mathcal{L}_{D 4},
\end{aligned}
$$

where

$$
\begin{aligned}
& \tau_{4}=\mu_{4} \Omega_{3} g_{s}^{-1} R^{3} \frac{U_{0}}{2^{2 / 3}}=\frac{N_{c} U_{0}}{2^{8 / 3}\left(2 \pi l_{s}^{2}\right)} \\
& \tilde{F}=\frac{2 \pi \alpha^{\prime} 2^{4 / 6}}{U_{0}} F_{t \theta}, \quad \tilde{A}_{t}=\frac{2^{2 / 3}}{U_{0}} \cdot 2 \pi \alpha^{\prime} A_{t} .
\end{aligned}
$$

The dimensionless displacement can be defined as follows;

$$
\begin{aligned}
\frac{\partial \mathcal{L}_{D 4}}{\partial \tilde{F}} & =\frac{\sin ^{3} \theta \tilde{F}}{\sqrt{\left(\omega_{-}^{2} / \omega_{+}^{2 / 3}\right)\left(\xi^{2}+\xi^{\prime 2}\right)-\tilde{F}^{2}}} \\
& \equiv-D(\theta) .
\end{aligned}
$$

Then the equation of motion for gauge field is

$$
\partial_{\theta} D(\theta)=-3 \sin ^{3} \theta .
$$

Integrating, we get

$$
D(\theta)=2(2 \nu-1)+3\left(\cos \theta-\frac{1}{3} \cos ^{3} \theta\right),
$$

where the integration constant $\nu$ determines the number of fundamental sting ( $\nu N_{c}$ strings are attached at south pole and $(1-\nu) N_{c}$ strings at north pole, we set $\left.\nu=0\right)$ 
By performing Legendre transformation, we can get 'Hamiltonian'

$$
\begin{aligned}
\mathcal{H}_{D 4} & =\tilde{F} \frac{\partial \mathcal{L}_{D 4}}{\partial \tilde{F}}-\mathcal{L}_{D 4} \\
& =\tau_{4} \int d \theta \sqrt{\frac{\omega_{-}^{2}}{\omega_{+}^{2 / 3}}\left(\xi^{2}+\xi^{\prime 2}\right)} \sqrt{D(\theta)^{2}+\sin ^{6} \theta}
\end{aligned}
$$

The numerical solution of the equation of motion for above Hamiltonian is drawn at FIG. 2. There is no closed D4 brane solution which can be a baryon vertex, except a D4 brane wrapping blackhole horizon itself. We also get same results of absence of D5 baryon vertex in D3 deconfing background.

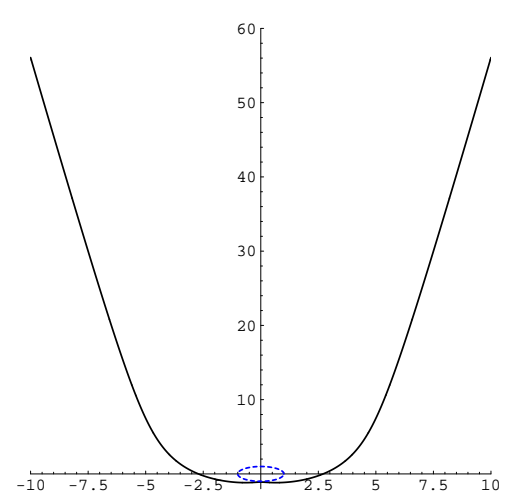

(a)

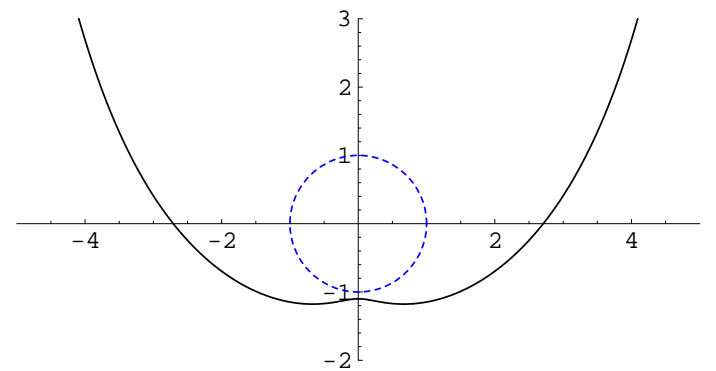

(b)

FIG. 2: (a) Solution of baryon D4 brane in deconfined phase. (b) Solution near the origin

Since there is no candidate for the baryon vertex, there is no point to discuss the baryon on the D6 brane theory. All the baryon charges exists as quarks (strings attached on it) and there are no Minkowski embedding in this background. This gravity result is consistent with that of gauge theory result in deconfined phase: there is no baryon in deconfined phase. The thermodynamical properties of D6 brane in the blackhole background was discussed recently in [16], so we do not discuss them here. 


\section{THE BARYON MASS IN MEDIUM IN CONFINING BACKGROUND}

The non-supersymmetric geometry for confining background of D4 in Euclidean signature is given by

$$
\begin{aligned}
d s^{2} & =\left(\frac{U}{R}\right)^{3 / 2}\left(\eta_{\mu \nu} d x^{\mu} d x^{\nu}+f(U) d x_{4}^{2}\right)+\left(\frac{R}{U}\right)^{3 / 2}\left(\frac{d U^{2}}{f(U)}+U^{2} d \Omega_{4}^{2}\right) \\
e^{\phi} & =g_{s}\left(\frac{U}{R}\right)^{3 / 4}, \quad F_{4}=\frac{2 \pi N_{c}}{\Omega_{4}} \epsilon_{4}, \quad f(U)=1-\left(\frac{U_{K K}}{U}\right)^{3}, \quad R^{3}=\pi g_{s} N_{c} l_{s}^{3} .
\end{aligned}
$$

This background is related to the previous one by the double Wick rotation

$$
x_{4} \longleftrightarrow t, \quad t \longleftrightarrow x_{4}, \quad U_{0} \longleftrightarrow U_{K K}
$$

The Kaluza-Klein mass is defined as the inverse radius of the $x_{4}$ direction: $M_{K K}=\frac{3}{2} \frac{U_{K K}^{1 / 2}}{R^{3 / 2}}$. While $U_{K K}, g_{s}, R$ are bulk parameters, $M_{M M}$ and $g_{Y M}^{2}$ is the parameter of the gauge theory. They can be related by

$$
g_{s}=\frac{\lambda}{2 \pi l_{s} N_{c} M_{K K}}, \quad U_{K K}=\frac{2}{9} \lambda M_{K K} l_{s}^{2}, \quad R^{3}=\frac{\lambda l_{s}^{2}}{2 M_{K K}}, \quad \lambda=g_{Y M}^{2} N_{c} .
$$

Introducing a same dimensionless coordinate $\xi$ as previous section, the bulk geometry becomes

$$
d s^{2}=\left(\frac{U}{R}\right)^{3 / 2}\left(d t^{2}+d \vec{x}^{2}+f(U) d x_{4}^{2}\right)+\left(\frac{R}{U}\right)^{3 / 2}\left(\frac{U}{\xi}\right)^{2}\left(d \xi^{2}+\xi^{2} d \Omega_{4}^{2}\right)
$$

and $U, \xi$ are related by $\left(\frac{U}{U_{K K}}\right)^{3 / 2}=\frac{1}{2}\left(\xi^{3 / 2}+\frac{1}{\xi^{3 / 2}}\right)$.

\section{A. Baryon vertex - D4 brane}

In confining phase, we consider same configuration of baryon D4 brane wrapping on $S^{4}$. The background metric (16) can be written as

$$
d s^{2}=\left(\frac{U}{R}\right)^{3 / 2}\left(d t^{2}+f d x_{4}^{2}+d \vec{x}^{2}\right)+R^{3 / 2} \sqrt{U}\left(\frac{d \xi^{2}}{\xi^{2}}+d \theta^{2}+\sin ^{2} \theta d \Omega_{3}^{2}\right)
$$


We are using same world volume coordinate of D4 brane as previous section. The induced metric on the compact D4 brane is

$$
d s_{D 4}^{2}=\left(\frac{U}{R}\right)^{3 / 2} d t^{2}+R^{3 / 2} \sqrt{U}\left[\left(1+\frac{\xi^{\prime 2}}{\xi^{2}}\right) d \theta^{2}+\sin ^{2} \theta d \Omega_{3}^{2}\right],
$$

and $\xi^{\prime}=\partial \xi / \partial \theta$. The DBI action for single D4 brane with $N_{c}$ fundamental string can be written as [17]

$$
\begin{aligned}
S_{D 4} & =-\mu_{4} \int e^{-\phi} \sqrt{\operatorname{det}\left(g+2 \pi \alpha^{\prime} F\right)}+\mu_{4} \int A_{(1)} \wedge G_{(4)} \\
& =\tau_{4} \int d t d \theta \sin ^{3} \theta\left[-\sqrt{\omega_{+}^{4 / 3}\left(\xi^{2}+\xi^{\prime 2}\right)-\tilde{F}^{2}}+3 \tilde{A}_{t}\right] \\
& =\int d t \mathcal{L}_{D 4},
\end{aligned}
$$

The Legendre transformed 'Hamiltonian' is

$$
\begin{aligned}
\mathcal{H}_{D 4} & =\tilde{F} \frac{\partial \mathcal{L}_{D 4}}{\partial \tilde{F}}-\mathcal{L}_{D 4} \\
& =\tau_{4} \int d \theta \sqrt{\omega_{+}^{4 / 3}\left(\xi^{2}+\xi^{\prime 2}\right)} \sqrt{D(\theta)^{2}+\sin ^{6} \theta}
\end{aligned}
$$

where displacement $D(\theta)$ is same as (11). Solutions of equation of motion for above Hamiltonian can be obtained numerically. We set $\nu=0$ because we assume that all fundamental strings are attached at north pole $(\theta=\pi)$. Then we should impose smooth boundary condition $\left(\xi^{\prime}(0)=0\right.$ and $\left.\xi(0)=\xi_{0}\right)$ at $\theta=0$. Numerical solutions are parameterized by initial value of $\xi_{0}$. The solutions corresponding to different $\xi_{0}$ 's are drawn in FIG. 3. In this figure, $N_{c}$ fundamental strings are attached at the cusp. This configuration will be stable only if there is tension balance between two object.

If we call the position of the cusp of $\mathrm{D} 4$ brane by $U_{c}$, the force from the $\mathrm{D} 4$ brane tension 


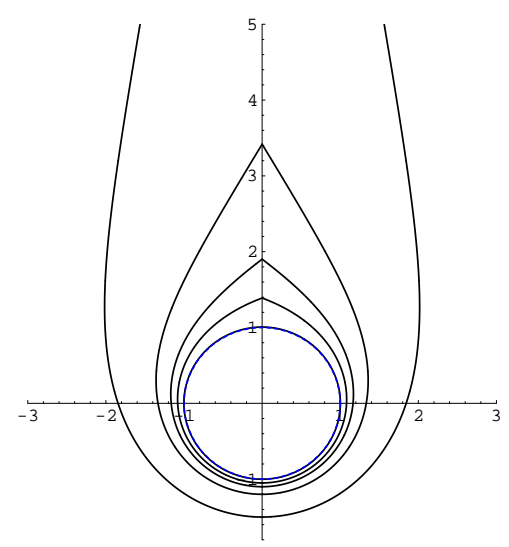

FIG. 3: Shape of D4 brane for different $\xi_{0}$.

can be obtained by varying the Hamiltonian of D4 brane with respect to $U_{c}$ while keeping other variables;

$$
\begin{aligned}
F_{D 4} & =\left.\frac{\partial \mathcal{H}}{\partial U_{c}}\right|_{\text {fix other values }} \\
& =N_{c} T_{F}\left(\frac{1+\xi_{c}^{-3}}{1-\xi_{c}^{-3}}\right) \frac{\xi_{c}^{\prime}}{\sqrt{\xi_{c}^{2}+\xi_{c}^{\prime \prime}}},
\end{aligned}
$$

where $T_{F}=\frac{2^{2 / 3} \tau_{4}}{N_{c} U_{K K}}$ is tension of fundamental string. The tension of D4 brane is always smaller or equal to the fundamental string. Therefore, if there were no probe branes, the cusp should be pulled up to infinity and the final configuration of D4 would be 'tube-like' shape as in [18]. So far we considered single compact D4 brane with $N_{c}$ fundamental strings. Now we need to study the deformation of the D6 which is pulling the compact D4 brane by fundamental strings.

\section{B. Fundamental strings connecting baryon vertex and the probe brane}

If we take D4-D6 system with confining background and consider the baryon vertex as compact D4, both D4 and D6 deform due to the interaction between them through fundamental string (F1) and the D4 background. When strings and D-branes are in contact, the length of the F1 tends to be zero while the D-branes are deformed to replace them for minimum energy configuration. One may say that "F1 is the most expensive object of all branes". Therefore the strings connecting the D4 and D6 have zero length and the D4 
and D6 are contacting at a point. In fact there is no way to connect D-branes of different dimensionality by a tube-like deformation even before we consider a solution of a DBI system. The point contact is the only possible configuration.

\section{Probe D6 brane}

Now, we put probe D6 brane where the other end points of fundamental strings are attached. The string endpoints can be understood as point charges on D6 brane. The gauge potential which couples to this point source is $A_{t}$. The the bulk metric (16) can be written as

$$
d s^{2}=\left(\frac{U}{R}\right)^{3 / 2}\left(d t^{2}+d \vec{x}^{2}+f(U) d x_{4}^{2}\right)+\left(\frac{R}{U}\right)^{3 / 2}\left(\frac{U}{\xi}\right)^{2}\left(d \rho^{2}+\rho^{2} d \Omega_{2}^{2}+d y^{2}+y^{2} d \phi^{2}\right)
$$

where D6 brane world volume coordinates are $\left(t, \vec{x}, \rho, \theta_{\alpha}\right)$. The embedding ansatz is that only $y$ depends on $\rho$ and $\phi=0$. The induced metric on D6 brane is

$$
d s_{D 6}^{2}=\left(\frac{U}{R}\right)^{3 / 2}\left(d t^{2}+d \vec{x}^{2}\right)+\left(\frac{R}{U}\right)^{3 / 2}\left(\frac{U}{\xi}\right)^{2}\left[\left(1+\dot{y}^{2}\right) d \rho^{2}+\rho^{2} d \Omega_{2}^{2}\right]
$$

where $\dot{y}=\partial y / \partial \rho$.

The DBI action for $N_{f}$ D6 brane is

$$
\begin{aligned}
S_{D 6}=\int d t \mathcal{L}_{D 6} & =-N_{f} \mu_{6} \int e^{-\phi} \sqrt{\operatorname{det}\left(g+2 \pi \alpha^{\prime} F\right)} \\
& =-\tau_{6} \int d t d \rho \rho^{2} \omega_{+}^{4 / 3} \sqrt{\omega_{+}^{4 / 3}\left(1+\dot{y}^{2}\right)-\tilde{F}^{2}}
\end{aligned}
$$

where

$$
\tau_{6}=\frac{1}{4} N_{f} \mu_{6} V_{3} \Omega_{2} g_{s}^{-1} U_{K K}^{3}, \quad \tilde{F}=\frac{2 \cdot 2^{2 / 3} \pi \alpha^{\prime} F_{t \rho}}{U_{K K}}
$$

We define dimensionless quantity $\tilde{Q}$ from the equation of motion for $\tilde{F}$;

$$
\frac{\partial S_{D 6}}{\partial \tilde{F}}=\frac{\rho^{2} \omega_{+}^{4 / 3} \tilde{F}}{\sqrt{\omega_{+}^{4 / 3}\left(1+\dot{y}^{2}\right)-\tilde{F}^{2}}} \equiv \tilde{Q}
$$


The hamiltonian is connected to the number of point sources (number of fundamental strings) $Q$ by

$$
\tilde{Q}=\frac{U_{K K} Q}{2 \cdot 2^{2 / 3} \pi \alpha^{\prime} \tau_{6}} .
$$

The 'Hamiltonian' can be obtained by Legendre transformation;

$$
\begin{aligned}
\mathcal{H}_{D 6} & =\tilde{F} \frac{\partial S_{D 6}}{\partial \tilde{F}}-S_{D 6} \\
& =\tau_{6} \int d \rho \sqrt{\omega_{+}^{4 / 3}\left(\tilde{Q}^{2}+\rho^{4} \omega_{+}^{8 / 3}\right)} \sqrt{1+\dot{y}^{2}} \\
& =\tau_{6} \int d \rho V(\rho) \sqrt{1+\dot{y}^{2}}
\end{aligned}
$$

The equation of motion after eliminating the gauge field is written explicitly by

$$
\frac{\ddot{y}}{1+\dot{y}^{2}}+\frac{\partial \log V}{\partial \rho} \dot{y}-\frac{\partial \log V}{\partial y}=0
$$

and the Gauss-law constraint gives

$$
\tilde{F}=\frac{\tilde{Q} \omega_{+}^{4 / 3} \sqrt{1+\dot{y}^{2}}}{\sqrt{\tilde{Q}^{2}+\rho^{4} \omega_{+}^{8 / 3}}}
$$

We can solve the eqaution (30) if boundary condition is given: In $\tilde{Q}=0$ case, the solutions of probe D6 brane embedding are drawn in FIG. 4(a). Here we impose $\dot{y}(0)=0$ for the smoothness. The value of $y$ at the infinity corresponds to $m_{q}$. In $\tilde{Q} \neq 0$ case, there are fundamental strings which connect baryon D4 brane with probe D6 brane. In this case, force balance condition request that $\dot{y}(0)$ be non-zero. The force at the cusp of probe D6 brane can be obtained as;

$$
\begin{aligned}
\hat{F}_{D 6} & =\left.\frac{\partial \mathcal{H}_{D 6}}{\partial U_{c}}\right|_{\partial} \\
& =\frac{Q}{2 \pi \alpha^{\prime}}\left(\frac{1+\xi_{c}^{-3}}{1-\xi_{c}^{-3}}\right) \frac{\dot{y}_{c}}{\sqrt{1+\dot{y}_{c}^{2}}}
\end{aligned}
$$

The whole configuration (compact $\mathrm{D} 4+\mathrm{F} 1+\mathrm{D} 6)$ can be stationary if there is a force balance 


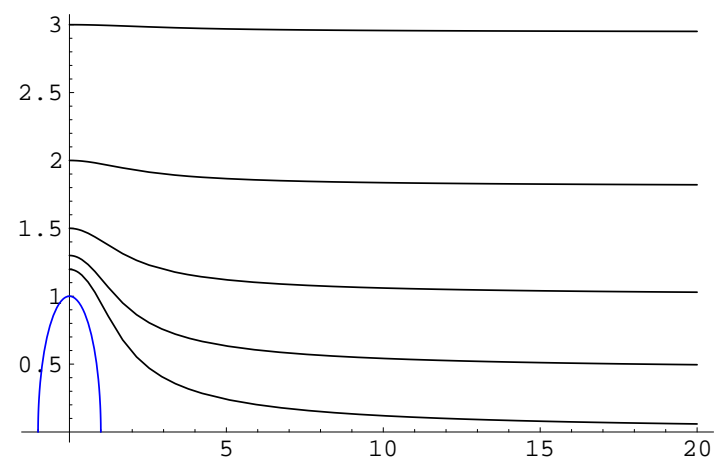

(a)

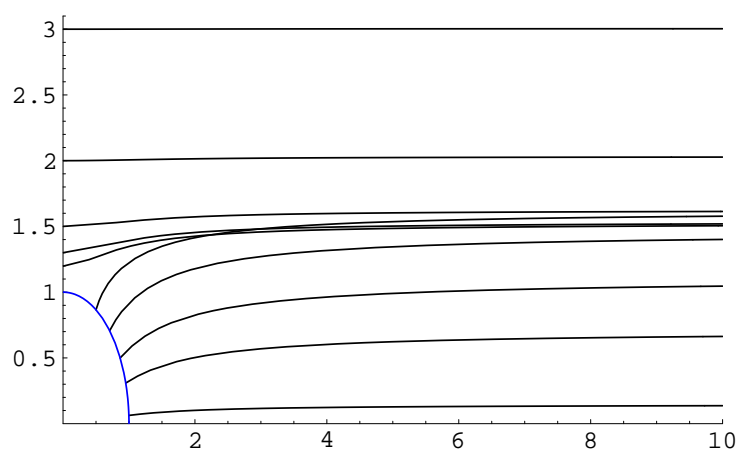

(b)

FIG. 4: D6 brane embedding with different $y_{0}$ in the case of $Q=0$. Blue circle denote singularity or horizon. (a) In confinement phase. (b) In deconfined phase

condition. For $Q$ fundamental strings, the number of baryon $\mathrm{D} 4$ is $Q / N_{c}$. The condition which makes the system to be stationary $\left(\rho=0, y_{c}=\xi_{c}\right)$ is:

$$
F_{D 6}=\frac{Q}{N_{c}} F_{D 4}
$$

which is simplified to be

$$
\dot{y}_{c}=\frac{\xi_{c}^{\prime}}{y_{c}} .
$$

With this, the value of $\dot{y}_{c}$ which satisfy force balance condition is uniquely determined for

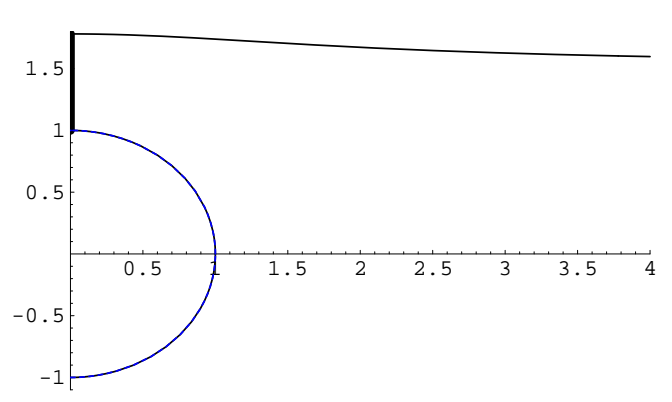

(a)

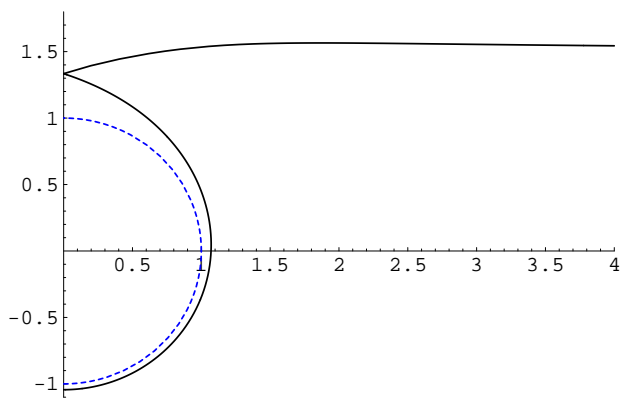

(b)

FIG. 5: Probe D6 brane embedding for $m_{q}=1.5$ (a)without force balance condition. Blue circle denote singularity where baryon D6 brane wrapped around. Thick vertical line denotes fundamental strings which are understood as a source. (b)with force balance condition.

given value of $\xi_{o}$ and $m_{q}$. The difference between the behaviors of probe D6 branes with and without the force balance condition is drawn in FIG. 5; In (a) we draw for the case Without 
FBC: $Q$ fundamental strings stretched between baryon D4 and probe D6 brane. (b) is for the case with FBC: baryon D4 brane is pulled up while probe D6 brane is pulled down such that the total system is stationary.

Since we established the existence of the baryon vertex, we now want to study how the the mass of baryon depends on the medium density. The difficulty comes from the broken symmetry: The very definition of the mass is the casimir Poincare invariance which is broken in the presence of the medium and it is not obvious what is the most natural definition of the baryon mass inside a medium.

As we discussed before, the length of the string of the baryon vertex is zero and therefore D4 and D6 are contacting each other at a point. There are two sources of contribution of the baryon mass change: One is the deformation of the compact D4 brane from the spherical shape and the other is the deformation of the probe D6 brane from the zero charge configuration. We believe that the latter is responsible for the baryon-baryon interaction while the former is related to the quark-quark interaction to form a baryon in the medium. Therefore we define the mass in the medium as the energy of the deformed compact D-brane. Then, the mass of a baryon is proportional to the value of $\xi_{0}$. The density dependence of a mass of baryon D4 brane for several value of $m_{q}$ is drawn in FIG. 6(a). For large current quark mass, the baryon mass decreases as a function of the density. On the other hand, for the small quark mass it has a minimum. Similar behavior was observed in D4/D8/D 8 system[15], where we have a zero current quark mass. See FIG. 6(b).

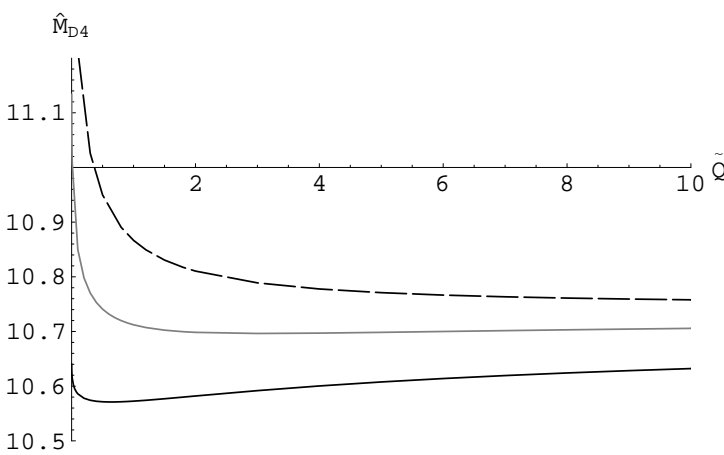

(a)

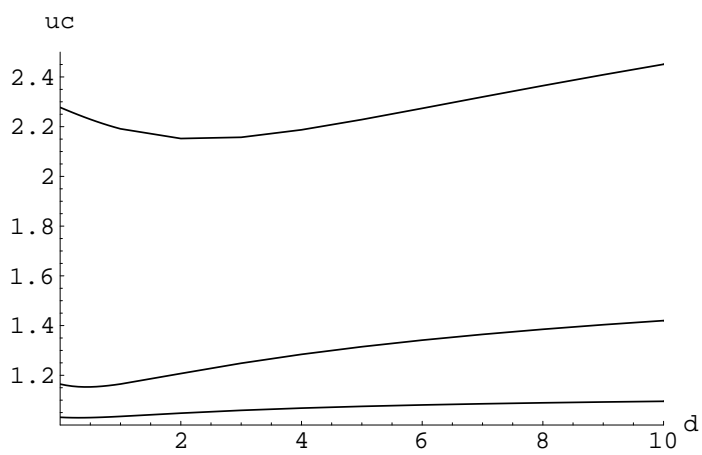

(b)

FIG. 6: (a) Baryon Mass in medium for D4/D6 system with current quark masses $m_{q}=1.5$ (dashed line), $m_{q}=1$ (gray line), $m_{q}=0$ (real line). (b) The position (mass) of D4 brane as a function of the electric displacement $\mathrm{d}$ for $l=0.5, l=1$ and $l=1.5$ from top to below $(l=1$ result is obtained in $[15])$. 


\section{Binding energy of baryons and their interaction energy}

Consider the configuration where baryon vertex and probe brane is not connected. The position of baryon vertex is lowest possible value $U_{K K}$ and the shape is spherical. See Fig. 5 (a). The energy of this configuration $\mathcal{F}^{(0)}$ is given by

$$
\begin{aligned}
\mathcal{F}^{(0)} & =\mathcal{H}_{D 6}^{(0)}+N_{B} \mathcal{H}_{D 4}^{(0)}+Q \mathcal{H}_{F 1} \\
& =\tau_{6}\left[\hat{\mathcal{H}}_{D 6}^{(0)}(\tilde{Q}=0)+\frac{\tilde{Q}}{4} \hat{\mathcal{H}}_{D 4}^{(0)}(\tilde{Q})+\tilde{Q} \int_{1}^{\xi_{c}^{0}}\left(1+\xi^{-3}\right)^{2 / 3} d \xi\right]
\end{aligned}
$$

where $\mathcal{H}_{D 6}^{(0)}$ is the probe D6 brane energy without charge, $\mathcal{H}_{D 4}^{(0)}$ is the compact and spherical D4 brane energy without attached string and $\xi_{c}^{0}$ is a position of D6 brane at $\rho=0$ and $N_{B}$ is a baryon number $Q / N_{c}$. The energy of configuration (b) in Fig. 5 with FBC imposed is given by

$$
\mathcal{F}=\tau_{6}\left[\hat{\mathcal{H}}_{D 6}(\tilde{Q})+\frac{\tilde{Q}}{4} \hat{\mathcal{H}}_{D 4}\right]
$$

We define the baryon mass in a medium as the energy of the deformed D4 with FBC imposed:

$$
M_{B}:=\mathcal{H}_{D 4}
$$

The binding energy of a baryon can be defined as the energy difference between the baryon vertices before and after the deformation:

$$
E_{B}=\mathcal{H}_{D 4}^{(0)}(Q)+N_{c} \mathcal{H}_{F 1}-M_{B}
$$

The baryon-baryon interaction energy can be defined as the deformation energy of the probe brane, that is, the difference between the energy of 'D6 without charge' and that of 'D6 with charge and FBC'. Since this energy present for any pair of the charges we expect that it is proportional to the $Q^{2}$ at least for small $\tilde{Q}$. Indeed the numerical result shows the quadratic dependence on the $Q$. When this quantity is positive (negative), baryon-baryon interaction is dominated by the repulsion(attraction).

The difference of $\mathcal{F}$ and $\mathcal{F}^{(0)}$ gives total binding energy plus interaction energy of the 
multi-baryon system:

$$
\mathcal{F}-\mathcal{F}^{(0)}=-N_{B} E_{B}+E_{\text {Interaction }}
$$

Numerical result of binding energy as well as the interaction energy is drawn in FIG. 7

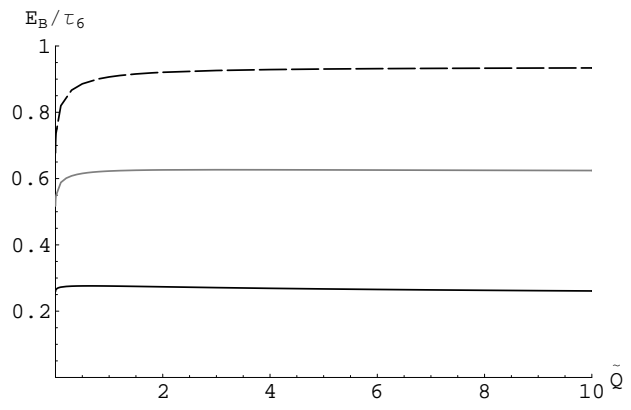

(a)

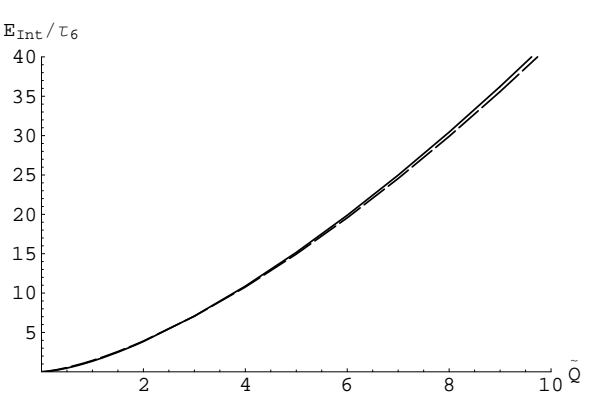

(b)

FIG. 7: (a) Binding energy for $m_{q}=0$ (real line), $m_{q}=1$ (gray line), $m_{q}=1.5$ (dashed line). (b) Interaction energy for $m_{q}=0$ (real line), $m_{q}=1.5$ (dashed line) $\left(m_{q}=1\right.$ line is almost same as dashed line)

One may ask what happen for D3 brane backgrounds with various probe branes. Naively we expect similar behavior in this case. Indeed for D3 brane background with D5 probe brane, completely parallel results are obtained. However, for D3/D7 system with one direction compactified, the results are not as expected. For the deconfining black hole background we do not have any baryon vertex configuration as expected. For the confining D3 background, there are two classes of embedding of D7 brane depending on whether D7 wraps the compact direction or not. In case D7 is wrapping the compact direction, the D7 does not get effectively repulsive force from the confining D3 background. In fact as far as D7 embedding is concerned, there is no difference in equation of motion between the black hole background and confining background, hence for small enough current quark mass, the D7 embedding should fall into the singularity, an impossible result. Therefore we do not expect that such D7 embedding is allowed. See FIG. 8(a). If D7 is not wrapping the compact direction, numerical study shows that no stable D7 configuration with finite current quark mass is allowed. See FIG. 8(b). We leave the description of some details to the appendix. 


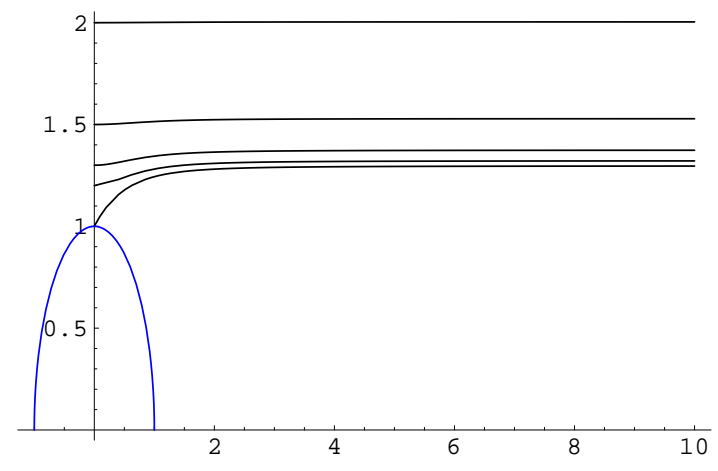

(a)

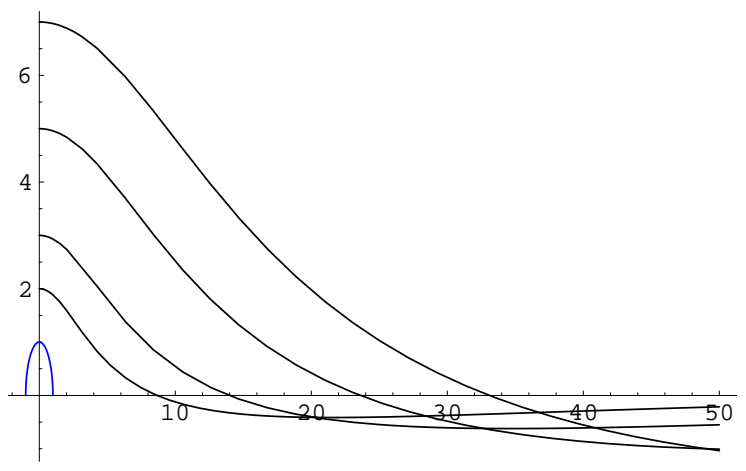

(b)

FIG. 8: D7 brane embedding with different $y_{0}$ in the case of $Q=0$. Blue circle denote minimal sphere in thermal $A d S_{5}$ : (a) When D7 wraps compactified spatial direction. (b) When D7 does not wraps compactified spatial direction.

\section{DISCUSSION}

We considered the issue of presence of Minkowski brane in the context of baryon mass in the medium. Although no analytic proof was made, our numerical investigation shows strong evidence of the absence of the baryon vertex operator in the black hole background. In the dual picture, this corresponds to the absence of the baryonic states in the $\mathrm{N}=2$ Yang Mills system with finite quark mass system. On the other hand, we found the existence of such states in the confining background like D3/D7 and D4/D6 double wick-rotation. Such picture is consistent with the naive expectations from the yang-Mills side that baryons exist only in the confining phase.

It is interesting to classify all the solution of the DBI action with source. The spiky solutions ( in deconfining background) represent quarks, while the cusp solutions (in confining) represent the baryons. Now, DBI action allows solutions which is regular everywhere. How should we interpret this solution in string theory setting? In fact, the very motivation of the Born-Infeld action is to regularize electromagnetic singularity at the point charge. The answer to this question is following: in the presence of dense medium, there are two scales in the system; one is the string scale controlling the scale of the spike and the other is the inter-particle distance. There are many order of magnitude difference between these scale. Therefore, the structure of one scale should be neglected when we treat that of the other scale. That is, when we focus on the physics of density, the spiky structure should be neglected. If we neglect the latter, then we get the smooth brane without singularity or cusp. 
This is the interpretation of the regular solution identified as the Minkowski embedding in [13]. However, we should not forget that there are invisible strings attached to the probe branes to give charge sources and their energy should be added to the total energy even though the shape of the regulated brane is smooth. In fact, if we add the string mass to Minkowski brane solution, the sum is bigger than that of the the black hole embedding, which means that none of the Minkowski embeddings in the quark phase are physically realized.

Notice that for the baryon phase also, the D5 mass should be always included as far as the baryonic states exist. In $\mathrm{QCD}$, most of the mass of the baryons are coming from the gluons instead of current quark mass. This has an analogue in hQCD: for the baryon vertex in gravity dual, the string length is always minimized and effectively strings are replaced by the deformed probe brane. This suggests that in hQCD, the quarks are melt away in confining phase and all the mass of the baryon is coming from the coherent gluons which is dual to the compact D-branes in the bulk.

Note added: After finishing this work, we received a relate work [19] where the the baryon vertex in the absence of the probe branes was discussed.

\section{APPENDIX A: D3 BRANE - CONFINING BACKGROUND}

We start usual Euclidean D3 black hole geometry.

$$
d s^{2}=\frac{U^{2}}{R^{2}}\left(d t^{2}+d \vec{x}^{2}+f(U) d \tau^{2}\right)+R^{2}\left(\frac{d U^{2}}{f(U) U^{2}}+d \Omega_{5}^{2}\right),
$$

where $R^{4}=2 \lambda l_{s}^{4}=4 \pi g_{s} N_{c} l_{s}^{4}$ and $f(U)=1-\left(U_{K K} / U\right)^{4}$. Here, $\lambda=g_{Y M}^{2} N_{c}$ is the 't Hooft coupling of the YM theory. $\tau$ direction is compactified with period

$$
\frac{1}{\beta_{\tau}}=\frac{U_{K K}}{\pi R^{2}}=\frac{M_{K K}}{2 \pi},
$$

where $M_{K K}$ is Kaluza-Klein mass. Introducing a dimensionless coordinate $\xi$ defined by $d \xi^{2} / \xi^{2}=d U^{2} /\left(U^{2} f(U)\right)$, the bulk geometry becomes

$$
d s^{2}=\frac{U^{2}}{R^{2}}\left(d t^{2}+f d \tau^{2}+d \vec{x}^{2}\right)+\frac{R^{2}}{\xi^{2}}\left(d \xi^{2}+\xi^{2} d \Omega_{5}^{2}\right),
$$


where $U$ and $\xi$ are related by $U^{2} / U_{K K}^{2}=\frac{1}{2}\left(\xi^{2}+1 / \xi^{2}\right)$ and $f=\left(1-\xi^{4}\right)^{2} /\left(1+\xi^{4}\right)^{2}$.

From now on, we will use the metric (A3) as a background metric with baryon vertex and probe brane.

\section{Baryon vertex - D5 brane}

The brane configuration which corresponds to the baryon vertex can be understood as D5 brane wrapping compact $S^{5}$ direction. The background metric (A3) can be written as

$$
d s^{2}=\frac{U^{2}}{R^{2}}\left(d t^{2}+f d \tau^{2}+d \vec{x}^{2}\right)+\frac{R^{2}}{\xi^{2}} d \xi^{2}+R^{2}\left(d \theta^{2}+\sin ^{2} \theta d \Omega_{4}^{2}\right),
$$

We assume that only $\xi$ depends on $\theta$ and $F_{t \theta} \neq 0$. The induced metric on D5 brane is

$$
d s_{D 5}^{2}=\frac{U^{2}}{R^{2}} d t^{2}+R^{2}\left(1+\frac{\xi^{\prime 2}}{\xi^{2}}\right) d \theta^{2}+R^{2} \sin ^{2} \theta d \Omega_{4}^{2} .
$$

The DBI action for single D5 brane with $N_{c}$ fundamental string can be written as similar to $[17]$

$$
\begin{aligned}
S_{D 5} & =-\mu_{5} \int \sqrt{\operatorname{det}\left(g+2 \pi \alpha^{\prime} F\right)}+\mu_{5} \int A_{(1)} \wedge G_{(5)} \\
& =\tau_{5} \int d t d \theta \sin ^{4} \theta\left[-\sqrt{\omega_{+}\left(\xi^{2}+\xi^{\prime 2}\right)-\tilde{F}^{2}}+4 \tilde{A}\right],
\end{aligned}
$$

where

$$
\tau_{5}=\frac{\mu_{5} \Omega_{4} R^{4} U_{K K}}{\sqrt{2}}, \quad \tilde{F}=\frac{2 \sqrt{2} \pi \alpha^{\prime} F_{t \theta}}{U_{K K}}, \quad \tilde{A}_{t}=\frac{\sqrt{2} \cdot 2 \pi \alpha^{\prime}}{U_{K K}} A_{t} .
$$

and $\xi^{\prime}=\partial \xi / \partial \theta$.

The displacement can be defined as follows;

$$
\frac{\partial \mathcal{L}_{D 5}}{\partial \tilde{F}}=\frac{\sin ^{4} \theta \tilde{F}}{\sqrt{\omega_{+}\left(\xi^{2}+\xi^{\prime 2}\right)-\tilde{F}^{2}}} \equiv-D(\theta) .
$$

From the equation of motion for gauge field, we can obtain

$$
D(\theta)=\left[\frac{3}{2}(\nu \pi-\theta)+\frac{3}{2} \sin \theta \cos \theta+\sin ^{3} \theta \cos \theta\right],
$$


where $\nu$ determines the number of fundamental sting $\left(\nu N_{c}\right.$ strings are at south pole and $(1-\nu) N_{c}$ strings are at north pole, we set $\left.\nu=0\right)$

By performing Legendre transformation, we can get 'Hamiltonian'

$$
\begin{aligned}
\mathcal{H}_{D 5} & =\tilde{F} \frac{\partial \mathcal{L}_{D 5}}{\partial \tilde{F}}-\mathcal{L}_{D 5} \\
& =\tau_{5} \int d \theta \sqrt{\omega_{+}\left(\xi^{2}+\xi^{\prime 2}\right)} \sqrt{D(\theta)^{2}+\sin ^{8} \theta}
\end{aligned}
$$

Solutions of equation of motion for above Hamiltonian are characterized by initial value of $\xi_{0}$ at $\theta=0$ (we set $\xi^{\prime}(\theta=0)=0$ as a initial condition. The solutions for different $\xi_{0}$ are qualitatively same as FIG. 3.

The force at the cusp of D5 brane tension is

$$
\begin{aligned}
F_{D 5} & =\left.\frac{\partial \mathcal{H}}{\partial U_{c}}\right|_{\text {fix other values }} \\
& =N_{c} T_{F}\left(\frac{1+\xi_{c}^{-4}}{1-\xi_{c}^{-4}}\right) \frac{\xi_{c}^{\prime}}{\sqrt{\xi_{c}^{2}+\xi_{c}^{\prime 2}}}
\end{aligned}
$$

where $T_{F}$ is tension of fundamental string. The force is always smaller than the tension of fundamental string same as baryon D4 brane case. Therefore, we have to find stable configuration with probe D-branes which make hole system to be stable.

\section{Probe D5 brane}

We put $N_{f}$ number of D5 branes as probe flavor brane. The background metric (A3) can be written as

$$
d s^{2}=\frac{U^{2}}{R^{2}}\left(d t^{2}+f d \tau^{2}+d x_{2}^{2}+d x_{3}^{2}\right)+\frac{R^{2}}{\xi^{2}}\left(d y^{2}+d \rho^{2}+\rho^{2} d \Omega_{2}^{2}+d z^{2}+z^{2} d \phi^{2}\right),
$$

where D5 brane world volume coordinates are $\left(t, x_{2}, x_{3}, \rho, \tau_{\alpha}\right)$. We also assume that the only coordinate $y$ depends on $\rho$ and focus on $z=\phi=0$ solution. The induced metric on D5 brane is

$$
d s_{D 5}^{2}=\frac{U^{2}}{R^{2}}\left(d t^{2}+d x_{2}^{2}+d x_{3}^{2}\right)+\frac{R^{2}}{\xi^{2}}\left[\left(1+\dot{y}^{2}\right) d \rho^{2}+\rho^{2} d \Omega_{2}^{2}\right]
$$


with non-zero electric field on it $\left(F_{\rho t} \neq 0\right)$ and $\dot{y}=\partial y / \partial \rho$.

DBI action of probe D5 brane is

$$
\hat{S}_{D 5}=-\hat{\tau}_{5} \int d t d \rho \rho^{2} \omega_{+} \sqrt{\omega_{+}\left(1+\dot{y}^{2}\right)-\tilde{F}^{2}} \equiv \int d t \hat{\mathcal{L}}_{D 5}
$$

where

$$
\hat{\tau}_{5}=\frac{1}{2 \sqrt{2}} N_{f} \mu_{5} \Omega_{2} V_{2} U_{K K}^{3}, \quad \tilde{F}=\frac{2 \sqrt{2} \pi \alpha^{\prime} F_{\rho t}}{U_{K K}} .
$$

We define dimensionless value $\tilde{Q}$ from equation of motion for $\tilde{F}$ is

$$
\frac{\partial \hat{S}_{D 5}}{\partial \tilde{F}}=\frac{\rho^{3} \omega_{+} \tilde{F}}{\sqrt{\omega_{+}\left(1+\dot{y}^{2}\right)-\tilde{F}}} \equiv \tilde{Q}
$$

The Legendre transformed 'Hamiltonian' from is

$$
\hat{\mathcal{H}}_{D 5}=\hat{\tau}_{5} \int d \rho \sqrt{\omega_{+}\left(\tilde{Q}^{2}+\rho^{4} \omega_{+}^{2}\right)} \sqrt{1+\dot{y}^{2}}
$$

with

$$
\tilde{Q}=\frac{U_{K K} Q}{2 \pi \alpha^{\prime} \sqrt{2} \hat{\tau}_{5}}
$$

where $Q$ is total number of quarks.

In $\tilde{Q}=0$ case, the solutions of probe D5 brane embedding are same as FIG. 4. In $\tilde{Q} \neq 0$ case, we can impose force balance condition

$$
\hat{F}_{D 5}=\frac{Q}{N_{c}} F_{D 5}
$$

with following constraint

$$
\dot{y}_{c}=\frac{\xi_{c}^{\prime}}{y_{c}}
$$

The D-brane configurations with and without FBC and the density dependence of baryon mass are qualitatively same as FIG. 5 and FIG. 6(a). 


\section{Probe D7 brane}

In this section, we consider D7 brane configuration used in $[13,20]$. In this case the background metric (A3) can be written as

$$
d s^{2}=\frac{U^{2}}{R^{2}}\left(d t^{2}+f d \tau^{2}+d x_{2}^{2}+d x_{3}^{2}\right)+\frac{R^{2}}{\xi^{2}}\left(d y^{2}+d \rho^{2}+\rho^{2} d \Omega_{3}^{2}+y^{2} d \phi^{2}\right)
$$

where $\xi^{2}=y^{2}+\rho^{2}$. D7 brane wraps compactified direction $\tau$. We also assume that the only coordinate $y$ depends on $\rho$. The induced metric on D7 brane is

$$
d s_{D 7}^{2}=\frac{U^{2}}{R^{2}}\left(d t^{2}+f d \tau^{2}+d x_{2}^{2}+d x_{3}^{2}\right)+\frac{R^{2}}{\xi^{2}}\left[\left(1+\dot{y}^{2}\right) d \rho^{2}+\rho^{2} d \Omega_{3}^{2}\right]
$$

with non-zero electric field on it $\left(F_{\rho t} \neq 0\right)$ and $\dot{y}=\partial y / \partial \rho$.

DBI action of D7 brane is

$$
S_{D 7}=-\tau_{7} \int d t d \rho \rho^{3} \omega_{-} \sqrt{\omega_{+}} \sqrt{\omega_{+}\left(1+\dot{y}^{2}\right)-\tilde{F}^{2}}
$$

where,

$$
\tau_{7}=\frac{1}{4} N_{f} \mu_{7} \Omega_{3} \beta_{\tau} V_{2} U_{0}^{2}, \quad \tilde{F}=\frac{2 \sqrt{2} \pi \alpha^{\prime} F_{\rho t}}{U_{0}} .
$$

Equation of motion for $\tilde{F}$ is

$$
\frac{\partial \mathcal{L}_{D 7}}{\partial \tilde{F}}=\frac{\rho^{3} \omega_{-} \omega_{+}^{1 / 2} \tilde{F}}{\sqrt{\omega_{+}\left(1+\dot{y}^{2}\right)-\tilde{F}}} \equiv \tilde{Q}
$$

We can get a Hamiltonian from Legendre transformation,

$$
\mathcal{H}_{D 7}=\tau_{7} \int d \rho \sqrt{\omega_{+}\left(Q^{2}+\rho^{6} \omega_{-}^{2} \omega_{+}\right)} \sqrt{1+\dot{y}^{2}}
$$

In the case of $Q=0$, the Hamiltonian (A26) is same as the Hamiltonian for black hole background. However, in confining background, probe brane cannot end on the singularity. It means that probe brane cannot cover low $y_{\infty}$ region in $Q=0$ case. 


\section{Probe - D7 brane (II)}

Here we consider the probe D7 brane does not contain compact direction. The background metric (A3) can be written as

$$
d s^{2}=\frac{U^{2}}{R^{2}}\left(d t^{2}+f d \tau^{2}+d x_{2}^{2}+d x_{3}^{2}\right)+\frac{R^{2}}{\xi^{2}}\left(d y^{2}+d \rho^{2}+\rho^{2} d \Omega_{4}^{2}\right)
$$

where $\xi^{2}=y^{2}+\rho^{2}$. We also assume that only $y$ depends on $\rho$. The induced metric on D7 brane is

$$
d s_{D 7}^{2}=\frac{U^{2}}{R^{2}}\left(d t^{2}+d x_{2}^{2}+d x_{3}^{2}\right)+\frac{R^{2}}{\xi^{2}}\left[\left(1+\dot{y}^{2}\right) d \rho^{2}+\rho^{2} d \Omega_{4}^{2}\right]
$$

with non-zero electric field on it $\left(F_{\rho t} \neq 0\right)$ and $\dot{y}=\partial y / \partial \rho$.

DBI action of D7 brane is

$$
S_{D 7}=-\tau_{7} \int d t d \rho \frac{\rho^{4} \omega_{+}}{\xi^{2}} \sqrt{\omega_{+}\left(1+\dot{y}^{2}\right)-\tilde{F}^{2}}
$$

where,

$$
\tau_{7}=\frac{1}{2 \sqrt{2}} N_{f} \mu_{7} \Omega_{4} V_{2} R^{2} U_{0}^{3}, \quad \tilde{F}=\frac{2 \sqrt{2} \pi \alpha^{\prime} F_{\rho t}}{U_{0}}
$$

Equation of motion for $\tilde{F}$ is

$$
\frac{\partial \mathcal{L}_{D 7}}{\partial \tilde{F}}=\frac{\rho^{4} \omega_{+} \tilde{F}}{\xi^{2} \sqrt{\omega_{+}\left(1+\dot{y}^{2}\right)-\tilde{F}}} \equiv \tilde{Q}
$$

We can get a Hamiltonian from Legendre transformation,

$$
\mathcal{H}_{D 7}=\tau_{7} \int d \rho \sqrt{\omega_{+}\left(Q^{2}+\frac{\rho^{8} \omega_{+}^{2}}{\xi^{4}}\right)} \sqrt{1+\dot{y}^{2}}
$$

As we discussed before, the solution of equation of motion for above Hamiltonian gives funny behavior even $Q=0$ case. D7 brane does not goes to flat brane as $y_{0}$ increase. Moreover, values of $y_{\infty}$ are very small for every $y_{0}$ even for a very large value of $y_{0}$. It means that the value of $y_{\infty}$ cannot cover whole $y$ axes. And therefore we do not consider this case seriously. 


\section{Acknowledgement}

We want to thank Shin Nakamura and Mannque Rho for useful discussion and comments.

We also like to thank Jonathan Shock for his careful reading and correcting an error in eq.11 of the first version. This work is supported by KOSEF Grant R01-2007-000-10214-0. The work of SJS is also supported by the Korea Research Foundation Grant funded by the Korean Government (MOEHRD, Basic Research Promotion Fund) (KRF-2007-314-C00052) and by the SRC Program of the KOSEF through the CQUEST grant R11-2005-021.

[1] J. M. Maldacena, Adv. Theor. Math. Phys. 2231 (1998), hep-th/9711200. S. S. Gubser, I. R. Klebanov and A. M. Polyakov, Phys. Lett. B428 105 (1998), hep-th/9802109; E. Witten, Adv. Theor. Math. Phys. 2253 (1998), hep-th/9802150; O. Aharony, S. S. Gubser, J. M. Maldacena, H. Ooguri, and Y. Oz, Phys. Rept. 323183 (2000).

[2] E. Witten, JHEP 9807, 006 (1998) [arXiv:hep-th/9805112].

[3] T. Sakai and S. Sugimoto, Prog. Theor. Phys. 113, 843 (2005) [arXiv:hep-th/0412141].

[4] T. Sakai and S. Sugimoto, Prog. Theor. Phys. 114, 1083 (2006) [arXiv:hep-th/0507073].

[5] K. Nawa, H. Suganuma and T. Kojo, Phys. Rev. D 75, 086003 (2007) [arXiv:hep-th/0612187].

[6] D. K. Hong, M. Rho, H. U. Yee and P. Yi, Phys. Rev. D 76, 061901 (2007) [arXiv:hepth/0701276].

[7] H. Hata, T. Sakai, S. Sugimoto and S. Yamato, "Baryons from instantons in holographic QCD," arXiv:hep-th/0701280.

[8] D. K. Hong, M. Rho, H. U. Yee and P. Yi, JHEP 0709, 063 (2007) [arXiv:0705.2632 [hep-th]].

[9] D. K. Hong, T. Inami and H. U. Yee, Phys. Lett. B 646, 165 (2007) [arXiv:hep-ph/0609270].

[10] J. Erlich, E. Katz, D. T. Son and M. A. Stephanov, Phys. Rev. Lett. 95261602 (2005), [arXiv:hep-ph/0501128];

L. Da Rold and A. Pomarol, Nucl. Phys. B721 79 (2005), [arXiv:hep-ph/0501218].

J. P. Shock, F. Wu, Y. L. Wu and Z. F. Xie, JHEP 0703 (2007) 064 [arXiv:hep-ph/0611227].

[11] K. Y. Kim, S. J. Sin and I. Zahed, "Dense hadronic matter in holographic QCD," [arXiv:hepth/0608046].

[12] N. Horigome and Y. Tanii, JHEP 0701, 072 (2007) [arXiv:hep-th/0608198].

[13] S. Nakamura, Y. Seo, S. J. Sin and K. P. Yogendran, "A new phase at finite quark density 
from AdS/CFT," [arXiv:hep-th/0611021].

[14] S. Kobayashi, D. Mateos, S. Matsuura, R. C. Myers and R. M. Thomson, JHEP 0702, 016 (2007) [arXiv:hep-th/0611099].

[15] O. Bergman, G. Lifschytz and M. Lippert, "Holographic Nuclear Physics," [arXiv:0708.0326].

[16] S. Matsuura, "On holographic phase transitions at finite chemical potential," [arXiv:0711.0407].

[17] C. G. . Callan, A. Guijosa, K. G. Savvidy and O. Tafjord, Nucl. Phys. B 555, 183 (1999) [arXiv:hep-th/9902197].

[18] C. G. . Callan and J. M. Maldacena, Nucl. Phys. B 513, 198 (1998) [arXiv:hep-th/9708147].

[19] K. Ghoroku and M. Ishihara, "Baryons with D5 Brane Vertex and k-Quarks," arXiv:0801.4216 [hep-th].

[20] S. Nakamura, Y. Seo, S. J. Sin and K. P. Yogendran, "Baryon-charge Chemical Potential in AdS/CFT," [arXiv:0708.2818]. 